\title{
Indications for contrast enhanced computed tomography in patients with sepsis induced acute kidney injury
}

\author{
Xinxin Chen \\ Department of Emergency Medicine, Affiliated Dongyang Hospital of Wenzhou Medical University, Dongyang 322100, China \\ Correspondence to: Dr. Xinxin Chen. Department of Emergency Medicine, Affiliated Dongyang Hospital of Wenzhou Medical University, Dongyang \\ 322100, China. Email: Chenxinxin0415@163.com.
}

Submitted Sep 24, 2019. Accepted for publication Sep 25, 2019.

doi: 10.21037/atm.2019.09.145

View this article at: http://dx.doi.org/10.21037/atm.2019.09.145

I read with great interest on the study by Miyamoto and colleagues (1). The study found no causal association between contrast media exposure and outcomes of sepsisinduced acute kidney injury (AKI). The study utilized sophisticated methodology of propensity score to adjust for the potential confounding by indication, minimizing the difference between patients with and without contrast media exposure. However, the pre-exposure conditions were not fully considered as that reported in the results. In clinical practice, the most important reasons for contrast media exposure during computed tomography (CT) in patients with sepsis-induced AKI is pancreatitis, because there is a large body of evidence showing that the contrast enhanced CT (CECT) is valuable in assessing the necrotic lesion of the pancreas (2). The most important cause of sepsis is pneumonia, but plain CT scan is usually enough for assessing infectious lesions of the lung. Thus, clinical indications for the CECT can provide important information on the difference between the two group of patients. The authors unfortunately did not provide such conditions in their original report. Secondly, it is unclear from the description (they described in the method section that: a CT scan was performed at admission) whether patients underwent plain CT on ICU admission but CECT on the other days was considered as the contrast group or as the control group. If they were considered as in the control group (plain CT scan on ICU admission), then their final outcome could be confounded by subsequent contrast media exposure (i.e., many patients may receive CECT because the first plain CT indicate abnormal conditions and need further confirmation). However, if they were considered as in the contrast group, the CECT can better be considered as a time-varying covariate that could be addressed with the time-dependent propensity score matching $(3,4)$. The evolution of a clinical conditions such as cancer and inflammatory diseases is considered to be important (5). Cross-sectional data usually fail to capture the temporal trend of the disease progression. Thus, more clarifications are mandatory for correct interpretation of the current results.

\section{Acknowledgments}

None.

\section{Footnote}

Conflicts of Interest: The author has no conflicts of interest to declare.

Ethical Statement: The author is accountable for all aspects of the work in ensuring that questions related to the accuracy or integrity of any part of the work are appropriately investigated and resolved.

\section{References}

1. Miyamoto Y, Iwagami M, Aso S, et al. Association between intravenous contrast media exposure and non-recovery from dialysis-requiring septic acute kidney injury: a nationwide observational study. Intensive Care Med 2019. [Epub ahead of print].

2. Huang H, Chen W, Tang G, et al. Optimal timing of contrast-enhanced computed tomography in an evaluation 
of severe acute pancreatitis-associated complications. Exp Ther Med 2019;18:1029-38.

3. Zhang Z, Reinikainen J, Adeleke KA, et al. Time-varying covariates and coefficients in Cox regression models. Ann Transl Med 2018;6:121.

4. Fisher LD, Lin DY. Time-dependent covariates in the Cox proportional-hazards regression model. Annu Rev Public Health 1999;20:145-57.

5. Qiu Z, Wu J, Wang Y, et al. Expression and clinical significance of negative costimulatory molecules B7-H1, B7-H3 and B7-H4 in the process of colorectal cancer's evolution. Transl Cancer Res 2018;7:1026-35.

Cite this article as: Chen X. Indications for contrast enhanced computed tomography in patients with sepsis induced acute kidney injury. Ann Transl Med 2019;7(20):597. doi: 10.21037/ atm.2019.09.145 